\title{
A STRONG LAW OF LARGE NUMBERS FOR HARMONIZABLE ISOTROPIC RANDOM FIELDS
}

\author{
RANDALL J. SWIFT \\ Western Kentucky University, Department of Mathematics \\ Bowling Green, KY 42101 USA \\ swiftr@wkuvx1.wku.edu
}

(Received September, 1996; Revised April, 1997)

\begin{abstract}
The class of harmonizable fields is a natural extension of the class of stationary fields. This paper considers a strong law of large numbers for the spherical average of a harmonizable isotropic random field.
\end{abstract}

Key words: Weakly and Strongly Harmonizable Fields, Isotropic, Nonstationary, MT Integrals, Laws of Large Numbers.

AMS subject classifications: $60 \mathrm{G} 12,60 \mathrm{G} 35$.

\section{Introduction}

Isotropic random fields play a key role in the statistical theory of turbulence. In addition to the assumption of isotropy, these fields have classically been considered stationary. However, there are applications under which the assumption of stationarity is not physically realistic, e.g. detection of a phase modulated signal. Harmonizable fields provide a natural extension to the stationary class by retaining the powerful Fourier analytic techniques while relaxing the assumption of stationarity.

This paper recalls the necessary theory of harmonizable isotropic random fields and obtains conditions for a strong law of large numbers to be valid for the spherical average of a harmonizable isotropic random field. Yadrenko [14] obtained a similar result for stationary isotropic random fields.

\section{Preliminaries}

To introduce the desired class of random functions, recall that if a random field $X: \mathbb{R}^{n} \rightarrow L_{0}^{2}(P)$ is stationary then it can be expressed as

$$
X(t)=\int_{\mathbb{R}^{n}} e^{i \lambda t} d Z(\lambda),
$$

where $Z(\cdot)$ is a $\sigma$-additive stochastic measure on the Borel $\sigma$-algebra $\mathscr{B}$ of $\mathbb{R}^{n}$, with orthogonal values in the complex Hilbert space, $L_{0}^{2}(P)$, of centered random variables. The covariance, $r(\cdot, \cdot)$, of the field is 


$$
r(s, t)=\int_{\mathbb{R}^{n}} e^{i(s-t) \lambda} d F(\lambda),
$$

where $E\left(Z(A) \overline{Z(B))}=F(A \cap B), F\right.$ a positive finite Borel measure on $\mathbb{R}^{n}$. Here $E(\cdot)$ denotes the expectation.

A generalization of the concept of stationarity is given by fields $X: \mathbb{R}^{n} \rightarrow L_{0}^{2}(P)$ with covariance $r(\cdot, \cdot)$ expressible as

$$
r(s, t)=\int_{\mathbb{R}^{n}} \int_{\mathbb{R}^{n}} e^{i \lambda s-i \lambda^{\prime} t} d F\left(\lambda, \lambda^{\prime}\right)
$$

where $F(\cdot, \cdot)$ is a complex bimeasure, called the spectral bimeasure of the field, of bounded variation in the Vatiali's sense or more inclusively in Fréchet's sense; in which case the integrals are strict Morse-Transue (cf. Rao [5] and Chang and Rao [1]). The covariance as well as the field are termed strongly or weakly harmonizable respectively. Every weakly or strongly harmonizable field $X: \mathbb{R}^{n} \rightarrow L^{2}(P)$ has an integral representation given by $(1)$, where $Z: \mathscr{B} \rightarrow L^{2}(P)$ is a stochastic measure (not necessarily with orthogonal values) and is called the spectral measure of the field. Both of these concepts reduce to the stationary case if $F$ concentrates on the diagonal $\lambda=\lambda^{\prime}$ of $\mathbb{R}^{n} \times \mathbb{R}^{n}$.

A subclass of random fields satisfy an additional condition called isotropy. Isotropic random fields $X(\cdot)$, have covariance $r(\cdot, \cdot)$ which are invariant under rotation and reflection. Isotropic fields play an important role in the statistical theory of turbulence, where direction in space is unimportant (cf. Yaglom [15]). Swift [10] obtained a representation of a weakly harmonizable isotropic covariance as

$$
r(s, t)=2^{\nu} \Gamma\left(\frac{n}{2}\right) \int_{0}^{\infty} \int_{0}^{\infty} \frac{J_{\nu}\left(\left\|\lambda s-\lambda^{\prime} t\right\|\right)}{\left\|\lambda s-\lambda^{\prime} t\right\|^{\nu}} d F\left(\lambda, \lambda^{\prime}\right)
$$

where $J_{\nu}(\cdot)$ is the Bessel function (of the first kind) of order $\nu=(n-2) / 2$ and $F(\cdot, \cdot)$ is a complex function of bounded Fréchet variation, with $\|\cdot\|$ denoting the vector norm.

Isotropic covariances $r(s, t)$ are functions of the lengths $\|s\|,\|t\|$ of the vectors $s, t$ and of the angle $\theta$ between $s$ and $t$. A representation in spherical-polar form for the covariances of harmonizable isotropic random fields was obtained by Swift as

$$
r(s, t)=\alpha_{n}^{2} \sum_{m=0}^{\infty} \sum_{l=1}^{h(m, n)} S_{m}^{l}(u) S_{m}^{l}(v) \int_{0}^{\infty} \int_{0}^{\infty} \frac{J_{m+\nu}\left(\lambda \tau_{1}\right) J_{m+\nu}\left(\lambda^{\prime} \tau_{2}\right)}{\left(\lambda \tau_{1}\right)^{\nu}\left(\lambda^{\prime} \tau_{2}\right)^{\nu}} d F\left(\lambda, \lambda^{\prime}\right)
$$
where

(i) $s=\left(\tau_{1}, u\right), t=\left(\tau_{2}, v\right)$ are the spherical polar coordinates of $s, t$ in $\mathbb{R}^{n}$, here $\tau_{1}=\|s\|, \tau_{2}=\|t\|$ and $u=s / \tau_{1}, v=t / \tau_{2}$ are unit vectors.

(ii) $\quad S_{m}^{l}(\cdot), 1 \leq l \leq h(m, n)=(2 m+2 \nu)(m+2 \nu-1) ! m !, m \geq 1, S_{0}^{l}(u)=1$ are the spherical harmonics on the unit $n$-sphere of order $m$.

(iii) $\quad \alpha_{n}>0, \alpha_{n}^{2}=2^{2 \nu+1} \Gamma\left(\frac{n}{2}\right) \pi^{\frac{n}{2}}$, with $F(\cdot, \cdot)$ as a complex function of bounded Fréchet variation.

Using (3) and Karhunen's Theorem, the spectral representation for a weakly har- 
monizable isotropic random field is given as

$$
X(t)=\alpha_{n} \sum_{m=0}^{\infty} \sum_{l=1}^{h(m, n)} S_{m}^{l}(u) \int_{0}^{\infty} \frac{J_{m+\nu}(\lambda \tau)}{(\lambda \tau)^{\nu}} d Z_{m}^{l}(\lambda)
$$

where $Z_{m}^{l}(\cdot)$ satisfies

$$
E\left(Z_{m}^{l}\left(B_{1}\right) \overline{Z_{m^{\prime}}^{l}\left(B_{2}\right)}\right)=\delta_{m m^{\prime}} \delta_{l l^{\prime}} F\left(B_{1}, B_{2}\right)
$$

with $F(\cdot, \cdot)$ a function of bounded Fréchet variation with the stochastic integral being in the Dunford-Schwartz sense, ([2], IV.10) and with $\delta_{m m^{\prime}}$ the Kronecker delta.

The theory of harmonizable random fields applicable to the statistical theory of turbulence is being developed by Rao, Swift, and others. The papers of Rao [7, 8] obtain representations for harmonizable isotropic random fields and their application to some sampling and prediction problems. The local behavior of some classes of harmonizable isotropic random fields has been considered by Swift [11-13]. Asymptotic properties of bispectral density estimators have recently been considered by H. Soedjak [9]. The book by Kakihara [3] gives a general treatment of multidimensional second order processes which include the harmonizable class.

\section{Laws of Large Numbers}

Let $X: \mathbb{R}^{n} \rightarrow L_{0}^{2}(P)$ be a strongly harmonizable isotropic random field and let $\gamma_{R}$ be the average over $B_{R}^{n}=\{t:\|t\| \leq R\}$, the ball of radius $R$ centered at the origin. Thus

$$
\begin{aligned}
\gamma_{R} & =\frac{1}{\operatorname{Vol}\left(B_{R}^{n}\right)} \int_{B_{R}^{n}} X(t) d t \\
& =\frac{n \alpha_{n}}{\sqrt{\omega_{n}} R^{n}} \int_{0}^{R} \tau^{n-1} \int_{0}^{\infty} \frac{J_{\nu}(\lambda \tau)}{(\lambda \tau)^{\nu}} d Z_{0}^{1}(\lambda) d \tau
\end{aligned}
$$

where (5) follows from (4) and the orthonormality of the spherical harmonics on $B_{R}^{n}$ (cf. Lebedev [4]). To obtain a law of large numbers for $\gamma_{R}$, one must show the variance of $\gamma_{R}$ is uniformly bounded, (cf. Rao [6]). Since $X(t)$ has zero mean, the variance of $\gamma_{R}$ is given as

$$
\begin{aligned}
E\left(\gamma_{R}^{2}\right) & =\frac{n^{2} \alpha_{n}^{2}}{\omega_{n} R^{2 n}} \int_{0}^{R} \int_{0}^{R} \tau_{1}^{n-1} \tau_{2}^{n-1} \int_{0}^{\infty} \int_{0}^{\infty} \frac{J_{\nu}\left(\lambda \tau_{1}\right) J_{\nu}\left(\lambda^{\prime} \tau_{2}\right)}{\left(\lambda \tau_{1}\right)^{\nu}\left(\lambda^{\prime} \tau_{2}\right)^{\nu}} d F\left(\lambda, \lambda^{\prime}\right) d \tau_{1} d \tau_{2} \\
& =\frac{n^{2} \alpha_{n}^{2}}{\omega_{n} R^{2 n}} \int_{0}^{\infty} \int_{0}^{\infty} \int_{0}^{R} \int_{0}^{R} \tau_{1}^{n / 2} \tau_{2}^{n / 2} \frac{J_{\nu}\left(\lambda \tau_{1}\right) J_{\nu}\left(\lambda^{\prime} \tau_{2}\right)}{\left(\lambda \lambda^{\prime}\right)^{\nu}} d \tau_{1} d \tau_{2} d F\left(\lambda, \lambda^{\prime}\right) .
\end{aligned}
$$

The relation (cf. Lebedev [4])

$$
\left|J_{\nu}(x)\right|<\frac{C_{\nu}}{x^{1 / 2}}
$$


which is valid for all $x$ with $C_{\nu}$ being a constant which only depends upon $\nu$, implies

$$
\begin{gathered}
\left|\int_{0}^{R} \tau^{n / 2} \frac{J_{\nu}(\lambda \tau)}{\lambda^{\nu}} d \tau\right| \leq \int_{0}^{R} \tau^{n / 2} \frac{\left|J_{\nu}(\lambda \tau)\right|}{\lambda^{\nu}} d \tau \\
\quad \leq \int_{0}^{R} \tau^{n / 2} \frac{C_{\nu}}{\lambda^{\nu}(\lambda \tau)^{1 / 2}} d \tau=\frac{2 C_{\nu} R^{\frac{n+1}{2}}}{(n+1) \lambda^{\frac{n-1}{2}}} .
\end{gathered}
$$

Using this expression together with (6) gives

$$
\begin{aligned}
& \left|E\left(\gamma_{R}^{2}\right)\right| \\
& \leq \frac{n^{2} \alpha_{n}^{2}}{\omega_{n} R^{2 n}} \int_{0}^{\infty} \int_{0}^{\infty} \int_{0}^{R} \int_{0}^{R} \tau_{1}^{n / 2} \tau_{2}^{n / 2} \frac{\left|J_{\nu}\left(\lambda \tau_{1}\right)\right|\left|J_{\nu}\left(\lambda^{\prime} \tau_{2}\right)\right|}{\left(\lambda \lambda^{\prime}\right)^{\nu}} d \tau_{1} d \tau_{2}\left|d F\left(\lambda, \lambda^{\prime}\right)\right| \\
& <\frac{n^{2} \alpha_{n}^{2}}{\omega_{n} R^{2 n}} \int_{0}^{\infty} \int_{0}^{\infty} \frac{C_{\nu} D_{\nu}}{\lambda^{\frac{n-1}{2}}\left(\lambda^{\prime}\right)^{\frac{n-1}{2}}} \frac{4 R^{\frac{n}{2}} R^{\frac{n}{2}}}{n^{2}}\left|d F\left(\lambda, \lambda^{\prime}\right)\right| \\
& =\frac{4 \alpha_{n}^{2} C_{\nu} D_{\nu} n^{2}}{\omega_{n} R^{n-1}(n+1)^{2}} \int_{0}^{\infty} \int_{0}^{\infty} \frac{\left|d F\left(\lambda, \lambda^{\prime}\right)\right|}{\left(\lambda \lambda^{\prime}\right)^{\frac{n-1}{2}}}
\end{aligned}
$$

where $C_{\nu}$ and $D_{\nu}$ are constants given by (7). Thus $\gamma_{R} \rightarrow 0$ in $L^{2}(P)$ as $R \rightarrow \infty$ if and only if

$$
\int_{0}^{\infty} \int_{0}^{\infty} \frac{\left|d F\left(\lambda, \lambda^{\prime}\right)\right|}{\left(\lambda \lambda^{\prime}\right)^{\frac{n-1}{2}}}<\infty .
$$

The preceding is summarized in the following proposition.

Proposition 3.1: The spherical average

$$
\gamma_{R}=\frac{1}{\operatorname{Vol}\left(B_{R}^{n}\right)} \int_{B_{R}^{n}} X(t) d t
$$

of a strongly harmonizable isotropic random field $X(\cdot)$ over $B_{R}^{n}=\{t:\|t\| \leq R\}$, the ball of radius $R$ centered at the origin, satisfies the weak law of large numbers if and only if

$$
\int_{0}^{\infty} \int_{0}^{\infty} \frac{\left|d F\left(\lambda, \lambda^{\prime}\right)\right|}{\left(\lambda \lambda^{\prime}\right)^{\frac{n-1}{2}}}<\infty
$$

There are many harmonizable isotropic random fields which satisfy condition (9), for instance, the field constructed by Swift [10]. In particular, Swift shows that if the spectral bimeasure $F(\cdot, \cdot)$ is absolutely continuous, with density $f$, having the specific form 


$$
f\left(\lambda, \lambda^{\prime}\right)=\frac{\omega_{n} \nu \lambda^{m+2 \nu+1}\left(\lambda^{\prime}\right)^{m+2 \nu+1}}{\alpha_{n}^{2}(m+\nu)} e^{\left(-\left(\lambda^{2}+\left(\lambda^{\prime}\right)^{2}\right)\right)}
$$

which is positive definite and where as before $\omega_{n}$ is the surface area of the unit sphere and $\nu=(n-2) / 2$, then

$$
r\left(\tau_{1}, \tau_{2}, \theta\right)=\left[1-2 \tau_{1} \tau_{2} \cos \theta+\tau_{1}^{2} \tau_{2}^{2}\right]^{-\nu} e^{\left(-\left(r_{1}^{2}+r_{2}^{2}\right)\right)}
$$

is the covariance of a strongly harmonizable isotropic random field. Using (10) with $m=1$ and simplifying (9) one obtains

$$
\int_{0}^{\infty} \int_{0}^{\infty} \frac{\left|d F\left(\lambda, \lambda^{\prime}\right)\right|}{\left(\lambda \lambda^{\prime}\right)^{\frac{n-1}{2}}}=\left(\Gamma\left(\frac{n+1}{4}\right)\right)^{2}<\infty .
$$

The condition (9) is also enough to guarantee that $\gamma_{R} \rightarrow \infty$ almost everywhere as $R \rightarrow \infty$, so that a strong law of large numbers prevails for the spherical average of a strongly harmonizable isotropic random field. In particular, the following theorem will now be shown.

Theorem 3.1: If a strongly harmonizable isotropic random field $X: \mathbb{R}^{n} \rightarrow L_{0}^{2}(P)$ has a spectral bimeasure $F(\cdot, \cdot)$ which satisfies the inequality $(9)$, then the spherical average $\gamma_{R}$ satisfies the strong law of large numbers.

Proof: The strong law of large numbers will first be shown to hold for a sequence of a radii $R_{k}=k^{\delta}$, where $0<\delta<1 /(3 n-1)$. Equation (8) implies that

$$
\sum_{k=1}^{\infty} E\left(\gamma_{R_{k}}^{2}\right)<\frac{4 \alpha_{n}^{2} C_{\nu} D_{\nu} n^{2}}{\omega_{n}(n+1)^{2}} \sum_{k=1}^{\infty} \frac{1}{k^{n \delta}}<\infty
$$

hence $\gamma_{R_{k}} \rightarrow 0$ almost everywhere as $k \rightarrow \infty$.

Now letting

$$
Y_{m}^{l}(\tau)=\alpha_{n} \int_{0}^{\infty} \frac{J_{m+\nu}(\lambda \tau)}{(\lambda \tau)^{\nu}} d Z_{m}^{l}(\lambda)
$$

and

one obtains

$$
d_{k}=\sup _{R_{k} \leq R \leq R_{k+1}}\left|\gamma_{R}-\gamma_{R_{k}}\right|
$$

$$
\begin{aligned}
& d_{k} \leq \frac{n}{\sqrt{\omega_{n}}}\left[\left(\frac{1}{R^{n}}-\frac{1}{R_{k}^{n}}\right) \int_{0}^{R_{k}} \tau^{n-1}\left|Y_{0}^{1}(\tau)\right| d \tau+\frac{1}{R^{n}} \int_{R_{k}}^{R} \tau^{n-1}\left|Y_{0}^{1}(\tau)\right| d \tau\right] \\
& \leq \frac{n}{\sqrt{\omega_{n}}}\left[\frac{R_{k+1}^{n}-R_{k}^{n}}{R_{k}^{2 n}} \int_{0}^{R_{k}} \tau^{n-1}\left|Y_{0}^{1}(\tau)\right| d \tau+\frac{1}{R_{k}^{n}} \int_{R_{k}}^{R_{k+1}} \tau^{n-1}\left|Y_{0}^{1}(\tau)\right| d \tau\right]
\end{aligned}
$$

Applying the elementary inequality $(a+b)^{2} \leq 2\left(a^{2}+b^{2}\right)$ gives

$$
d_{k}^{2} \leq \frac{2 n^{2}}{\omega_{n}^{2}}\left[\frac{\left(R_{k+1}^{n}-R_{k}^{n}\right)^{2}}{R_{k}^{4 n}} \int_{0}^{R_{k}} \int_{0}^{R_{k}} \tau_{1}^{n-1} \tau_{2}^{n-1}\left|Y_{0}^{1}\left(\tau_{1}\right)\right|\left|Y_{0}^{1}\left(\tau_{2}\right)\right| d \tau_{1} d \tau_{2}\right.
$$




$$
\left.+\frac{1}{R_{k}^{2 n}} \int_{0}^{R_{k}} \int_{0}^{R_{k}} \tau_{1}^{n-1} \tau_{2}^{n-1}\left|Y_{0}^{1}\left(\tau_{1}\right)\right|\left|Y_{0}^{1}\left(\tau_{2}\right)\right| d \tau_{1} d \tau_{2}\right]
$$

Now an application of the Cauchy-Buniakowski-Schwartz inequality yields

$$
\begin{gathered}
E\left|Y_{0}^{1}\left(\tau_{1}\right)\right|\left|Y_{0}^{1}\left(\tau_{2}\right)\right| \leq \alpha_{n}^{2} \sqrt{\left|\int_{0}^{\infty} \int_{0}^{\infty} \frac{J_{\nu}\left(\lambda \tau_{1}\right) J_{\nu}\left(\lambda^{\prime} \tau_{1}\right)}{\left(\tau_{1}^{2} \lambda \lambda^{\prime}\right)^{\nu}} d F\left(\lambda, \lambda^{\prime}\right)\right|} \\
\quad \sqrt[x]{\left|\int_{0}^{\infty} \int_{0}^{\infty} \frac{J_{\nu}\left(\lambda \tau_{2}\right) J_{\nu}\left(\lambda^{\prime} \tau_{2}\right)}{\left(\tau_{2}^{2} \lambda \lambda^{\prime}\right)^{\nu}} d F\left(\lambda, \lambda^{\prime}\right)\right|}
\end{gathered}
$$

but the inequality (7) implies

$$
\left|\int_{0}^{\infty} \int_{0}^{\infty} \frac{J_{\nu}\left(\lambda \tau_{1}\right) J_{\nu}\left(\lambda^{\prime} \tau_{1}\right)}{\left(\tau_{1}^{2} \lambda \lambda^{\prime}\right)^{\nu}} d F\left(\lambda, \lambda^{\prime}\right)\right|<\frac{C_{\nu} D_{\nu}}{\tau_{1}^{2 \nu+1 / 4} \tau_{2}^{\nu+1 / 4}} \int_{0}^{\infty} \int_{0}^{\infty} \frac{\left|d F\left(\lambda, \lambda^{\prime}\right)\right|}{\left(\lambda \lambda^{\prime}\right)^{\nu+1 / 2}}
$$

so that

$$
E\left|Y_{0}^{1}\left(\tau_{1}\right)\right|\left|Y_{0}^{1}\left(\tau_{2}\right)\right|<\frac{\alpha_{n}^{2} C_{\nu}^{1 / 2} D_{\nu}^{1 / 2}}{\tau_{1}^{\nu+1 / 4} \tau_{2}^{\nu+1 / 4}} \int_{0}^{\infty} \int_{0}^{\infty} \frac{\left|d F\left(\lambda, \lambda^{\prime}\right)\right|}{\left(\lambda \lambda^{\prime}\right)^{\nu+1 / 2}}
$$

Taking the expectation of (12) gives

$$
\begin{gathered}
E\left(d_{k}^{2}\right) \leq \frac{2 n^{2}}{\omega_{n}^{2}}\left[\frac{\left(R_{k+1}^{n}-R_{k}^{n}\right)^{2}}{R_{k}^{4 n}} \int_{0}^{R_{k}} \int_{0}^{R_{k}} \tau_{1}^{n-1} \tau_{2}^{n-1} E\left|Y_{0}^{1}\left(\tau_{1}\right)\right|\left|Y_{0}^{1}\left(\tau_{2}\right)\right| d \tau_{1} d \tau_{2}\right. \\
\left.+\frac{1}{R_{k}^{2 n}} \int_{0}^{R_{k}} \int_{0}^{R_{k}} \tau_{1}^{n-1} \tau_{2}^{n-1} E\left|Y_{0}^{1}\left(\tau_{1}\right)\right|\left|Y_{0}^{1}\left(\tau_{2}\right)\right| d \tau_{1} d \tau_{2}\right] \\
<M\left[\frac{\left(R_{k+1}^{n}-R_{k}^{n}\right)^{2}}{R_{k}^{4 n}} R_{k}^{3 / 2}+\frac{\left(R_{k+1}-R_{k}\right)^{3 / 2}}{R_{k}^{4 n}}\right]
\end{gathered}
$$

where $M$ is the finite constant given by

$$
M=\frac{2}{3} \alpha_{n}^{2} C_{\nu}^{1 / 2} D_{\nu}^{1 / 2} \int_{0}^{\infty} \int_{0}^{\infty} \frac{\left|d F\left(\lambda, \lambda^{\prime}\right)\right|}{\left(\lambda \lambda^{\prime}\right)^{\nu+1 / 2}}
$$

Replacing $R_{k}$ with $k^{\delta}$ and simplifying one obtains

$$
E\left(d_{k}^{2}\right)<\frac{A_{\delta}}{k^{(n-1) \delta}}+\frac{B_{\delta}}{k^{(3 n-1) \delta}}
$$

where $A_{\delta}$ and $B_{\delta}$ are finite constants which do not depend upon $k$. Hence 


$$
\sum_{k=1}^{\infty} E\left(d_{k}^{2}\right)<\infty
$$

so that $d_{k} \rightarrow 0$ almost everywhere as $k \rightarrow \infty$. But this implies $\gamma_{R} \rightarrow 0$ almost everywhere, as $R \rightarrow \infty$, proving the assertion.

\section{Acknowledgements}

The author expresses his thanks to Professor M.M. Rao for his continuing advice, encouragement and guidance during the work of this project. The author also expresses his gratitude to the Mathematics Department at Western Kentucky University for release time during the Spring 1996 semester, during which this work was completed.

\section{References}

[1] Chang, D.K. and Rao, M.M., Bimeasures and nonstationary processes, Real and Stochastic Analysis, John Wiley and Sons, New York (1986), 7-118.

[2] Dunford, N. and Schwartz, J.T., Linear Operators Part I, Interscience, New York 1957.

[3] Kakihara, Y., Multidimensional Second Order Stochastic Processes, World Scientific, Singapore 1997.

[4] Lebedev, N.N., Special Functions and Their Applications, Dover Publications, Inc., New York 1972.

[5] Rao, M.M., Harmonizable processes: Structure theory, L'Enseign Math 28 (1984), 295-351.

[6] Rao, M.M., Probability Theory with Applications, Academic Press, New York 1984.

[7] Rao, M.M., Sampling and prediction for harmonizable isotropic random fields, J. of Combinatorics, Info. and Sys. Sciences 16:2-3 (1991), 207-220.

[8] Rao, M.M., Characterization of isotropic harmonizable covariance and related representations, (preprint).

[9] Soedjak, H., Asymptotic Properties of Bispectral Density Estimators of Harmonizable Processes, Ph.D. Thesis, University of California, Riverside 1996.

[10] Swift, R.J., The structure of harmonizable isotropic random fields, Stoch. Anal. and Appl. 12 (1994), 583-616.

[11] Swift, R.J., A class of harmonizable isotropic random fields, $J$. of Combinatorics, Info. and Sys. Sciences 20:1-4 (1995), 111-127.

[12] Swift, R.J., Representation and prediction for locally harmonizable isotropic random fields, J. of Appl. Math and Stoch. Analysis 8:2 (1995), 101-114.

[13] Swift, R.J., Locally time-varying harmonizable spatially isotropic random fields, Indian J. of Pure and Appl. Math. 28:3 (1997), 295-310.

[14] Yadrenko, M.I., Spectral Theory of Random Fields, Optimization Software, Inc., New York (English Translation) 1983.

[15] Yaglom, A.M., Correlation Theory of Stationary and Related Random Functions, Vol. 1 and 2, Springer-Verlag, New York 1987. 


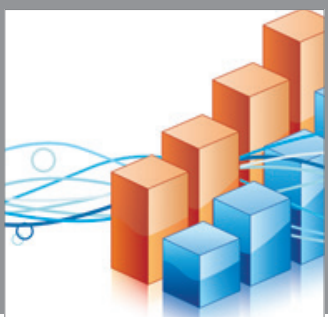

Advances in

Operations Research

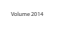

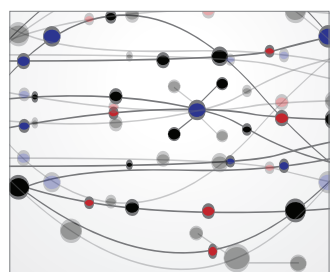

\section{The Scientific} World Journal
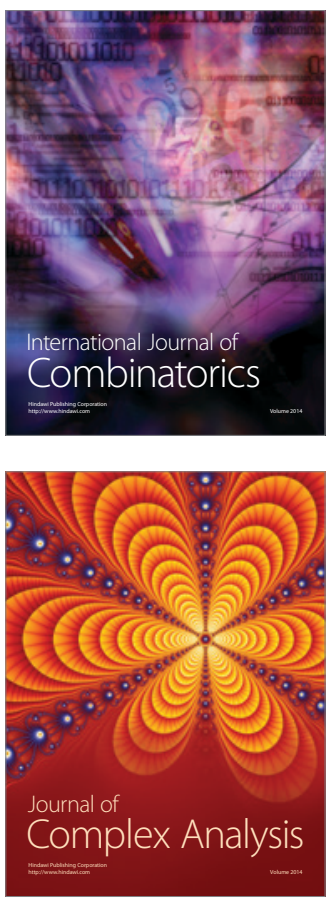

International Journal of

Mathematics and

Mathematical

Sciences
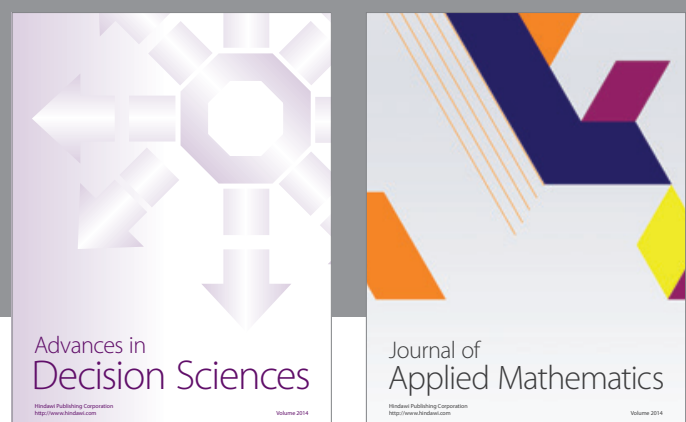

Journal of

Applied Mathematics
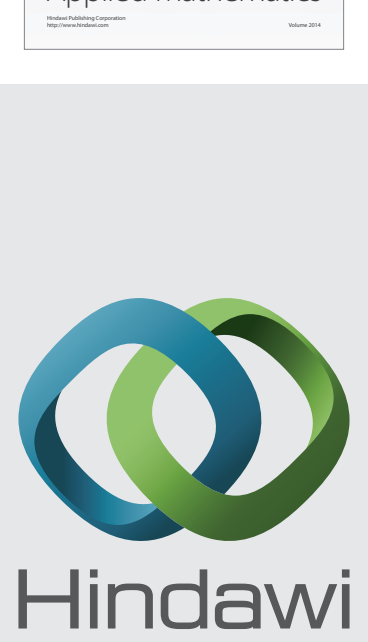

Submit your manuscripts at http://www.hindawi.com
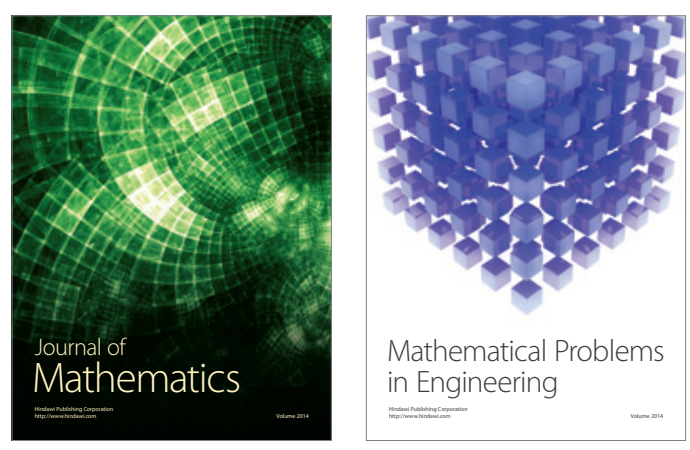

Mathematical Problems in Engineering
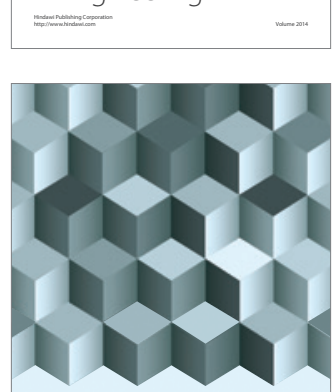

Journal of

Function Spaces
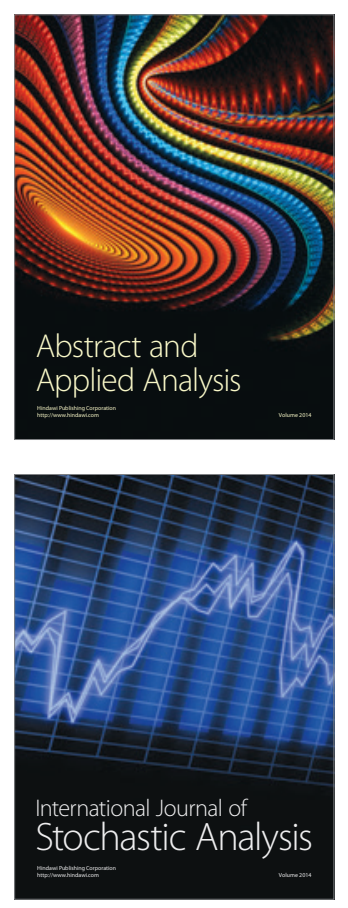

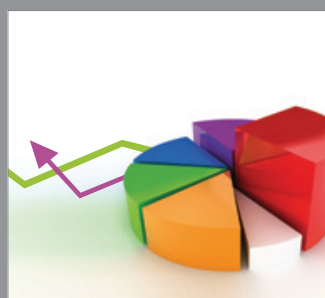

ournal of

Probability and Statistics

Promensencen
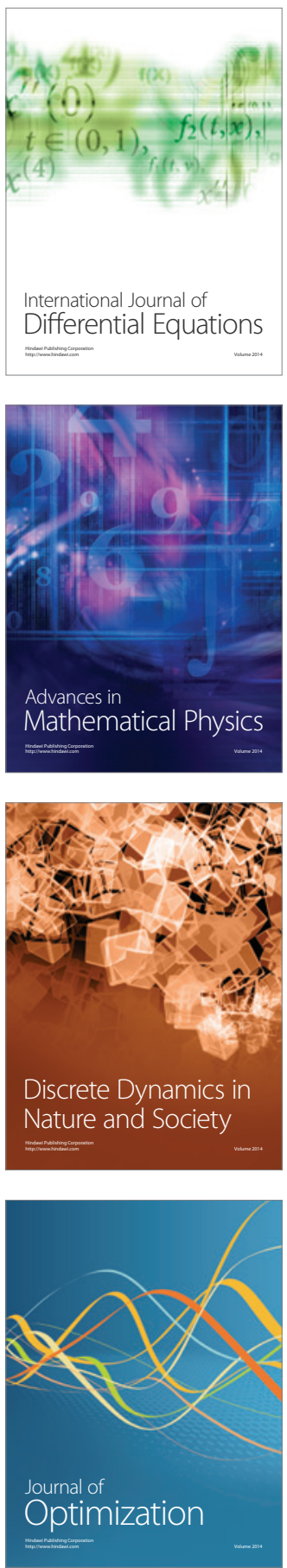